\title{
Understanding the Occurrence of a Wavy Wear Track on Elastomeric Materials
}

\author{
M. Khafidh ${ }^{1,2^{*}}$, B. Setiyana ${ }^{1,3}$, J. Jamari ${ }^{3}$, M.A. Masen ${ }^{4}$, D.J. Schipper ${ }^{1}$ \\ ${ }^{1}$ Faculty of Engineering Technology, University of Twente, P.O. Box 217, 7500AE, \\ Enschede, The Netherlands. \\ ${ }^{2}$ Dutch Polymer Institute DPI, P.O. Box 902, 5600AX Eindhoven, The Netherlands. \\ ${ }^{3}$ Department of Mechanical Engineering, University of Diponegoro, Jl. Prof. Soedharto SH, \\ Tembalang, Semarang 50275, Indonesia. \\ ${ }^{4}$ Department of Mechanical Engineering, Imperial College London, Exhibition Road, \\ London, SW7 2AZ, United Kingdom. \\ *email: m.khafidh@utwente.nl
}

\begin{abstract}
Elastomeric materials are used in daily applications, such as tires and conveyor belts. Sliding friction of elastomeric materials often produces a unique periodic wear pattern. In general, the periodic wear pattern has to be prevented because it generates surface instabilities and, as a result, vibration. Sliding contact between an elastomer and a rigid ball may cause the development of a periodic wear pattern like a wave on the elastomer surface, called a wavy wear track. The present study has investigated the occurrence of the wavy wear track on the elastomers under several operating conditions. The elastomers used were a Styrene-Butadiene Rubber (SBR) and Butadiene Rubber (BR) reinforced with two types of fillers, i.e., highly dispersible silica and short-cut aramid fiber. A pin-on-disc tribometer was used to study the wavy wear track of the materials under several operating conditions. An analytical model was used to predict the wave length of the wear pattern and to study the occurrence of the wavy wear track. The results show that the occurrence of the wavy wear track depends on the mechanical properties of the elastomer, the operating conditions (such as sliding velocity and force), the inertia mass of the counter surface frame and the circumferential length of the wear track. The analytical model is in good agreement with the experimental results.

Keywords: Styrene-Butadiene Rubber (SBR), Butadiene Rubber (BR), elastomer, sliding friction, wear, wavy wear track.
\end{abstract}




\section{Introduction}

Elastomeric materials are used in a wide range of applications, such as tires [1], wiper blades [2], conveyor belts [3] and seals [4]. In these applications, the elastomers contact with and slide against other components, which is a major source of frictional losses and wear. As a result, numerous investigations have been carried out regarding the sliding friction of elastomeric materials, focusing on topics such as the contact area [5], friction [6], wear [7] and lubrication [8]. Typically, materials that wear as the result of sliding contact with a harder counter material develop a fairly smooth, uniform wear track. However, elastomeric materials typically show a wear track that appears to have periodic features, as described in detail by [9]. In daily life, this phenomenon has been reported in several applications, such as tires [10] and conveyor belts [11]. Such a periodic pattern often generates surface instabilities at the elastomer surface. The resulting dynamics and fluctuations significantly reduce the performance of the rolling/sliding system, especially with respect to the positional accuracy, stability [12], vibration and noise [13]. Due to these disadvantages, the development of periodic wear patterns should be prevented in practical applications.

The surface irregularities that develop in a sliding elastomer contact often correlate with the so-called Schallamach waves [14]. Schallamach investigated the development of detachment waves in the sliding contact between soft elastomers and glass and found that a periodic pattern propagates across the contact zone from the leading edge to the trailing edge. Following the work of Schallamach, many studies have investigated the Schallamach waves, see for instance [15-20].

The development of periodic features and wear patterns have been investigated previously using needle [21-24] and blade [9, 25-29] indenters. Fukahori et al. [9, 29, 30] studied the periodic wear pattern between a blade indenter and an elastomer and observed a periodic crack pattern in the elastomer. They state that the initial cracks are formed due to micro-vibrations of the elastomer, followed by stick-slip oscillation that causes propagation of the cracks. The resulting spacing of the final pattern can be correlated with the stick-slip frequency.

In the case of a rigid sphere sliding against an elastomer counter surface, a macro wear pattern so-called wavy wear track may be created on the elastomer surface. This wavy wear track generates severe vibration in the system and furthermore influences both the frictional and wear behavior. Several experimental studies have been reported considering the stick-slip motion for the sliding contact between a rigid ball and an elastomer flat surface $[14,15,31$ - 
33]. Maegawa and Nakano [32] investigated the mechanism of stick-slip associated with Schallamach waves and found that stick-slip will occur at certain combinations of low forces and high sliding velocities. The Schallamach waves will generate variations in the contact area, which determines the stick-slip behavior, but the key parameters that influence the occurrence of a wavy wear track are not fully understood and, as a result, there is no method available to describe or predict the formation and the properties of the wavy wear track.

In the present study, the wavy wear track phenomenon of an elastomeric material was investigated. A pin-on-disc tribometer was used to study the occurrence of the wavy wear track, using a relatively large and smooth ball as the counter surface of the elastomeric flat. Two types of materials based on a Styrene-Butadiene Rubber (SBR) and Butadiene Rubber (BR) were used. A range of operating conditions was used to investigate the effects of the radius of sliding track, the sliding velocity and the normal force on the occurrence of a wavy wear track. A comparative study has been made between the analytical model and the experimental results.

\section{Materials and Methods}

\subsection{Materials}

Two types of elastomers were used in the present study: (1) a mixture of Styrene-Butadiene Rubber (SBR) and Butadiene Rubber (BR, high-cis-polybutadiene) with a formulation based on a silica-reinforced passenger car tire tread called "Green Tire” [34]. The rubber is reinforced with 80 phr (parts per hundred rubber) of highly dispersible silica using bis-(tri-ethoxy-silyl propyl) tetrasulphide (TESPT) as the coupling agent to provide a bond between the silica particles and the polymer; (2) a Styrene-Butadiene Rubber (SBR) reinforced with $15 \mathrm{phr}$ Aramid fibres supplied by Teijin Aramid B.V, Arnhem, The Netherlands. The initial length of the Aramid fibers is approximately $3 \mathrm{~mm}$, with a diameter of between 10 and $12 \mu \mathrm{m}$. A silane coupling agent NXT S-3-(triethoxysilylpropyl)-octanethioate was used to provide chemical bonding between the fibers and the matrix. The fibers were dispersed in random directions in the matrix, meaning that the mechanical properties of the material can be assumed to be the same in all directions and the material can be regarded as being isotropic. Details of the formulation in parts per hundred rubber (phr) are given in Table 1. 
Table 1. Material formulation of the elastomers.

\begin{tabular}{|c|c|c|c|}
\hline Ingredients & $\begin{array}{c}\text { SBR-BR } \\
\text { [in phr] }\end{array}$ & $\begin{array}{c}\text { SBR } \\
\text { [in phr] }\end{array}$ & Supplier \\
\hline $\begin{array}{l}\text { SBR, Buna VSL } \\
\text { VP PBR } 4045 \text { HM }\end{array}$ & - & 100 & Arlanxeo, Leverkusen, Germany \\
\hline $\begin{array}{l}\text { SBR, Buna VSL } \\
\text { 5025-2 HM }\end{array}$ & $97.3 *$ & - & Arlanxeo, Leverkusen, Germany \\
\hline BR, KBR 01 & 30.0 & - & Kumho, Seoul, S-Korea \\
\hline Silica Ultrasil VN3 & 80.0 & - & Evonik Industries AG, Essen, Germany \\
\hline Zinc oxide ( $\mathrm{ZnO})$ & 2.5 & 2.5 & Sigma Aldrich, St. Louis, United States \\
\hline Stearic acid (SA) & 2.5 & 1.5 & Sigma Aldrich, St. Louis, United States \\
\hline TDAE oil & 6.7 & - & $\begin{array}{l}\text { Hansen \& Rosenthal, Hamburg, } \\
\text { Germany }\end{array}$ \\
\hline $\begin{array}{l}\text { Twaron aramid } \\
\text { fiber }\end{array}$ & - & 15 & $\begin{array}{c}\text { Teijin Aramid B.V, Arnhem, } \\
\text { The Netherlands }\end{array}$ \\
\hline TESPT & 7.0 & - & Evonik Industries AG, Essen, Germany \\
\hline NXT & - & 6.0 & Momentive, New York, United States \\
\hline 6PPD & 2.0 & - & Flexsys, Brussels, Belgium \\
\hline TMQ & 2.0 & - & Flexsys, Brussels, Belgium \\
\hline Sulfur & 1.4 & 2.8 & Sigma Aldrich, St. Louis, United States \\
\hline N-Cyclohexyl & 1.7 & 3.4 & Flexsys, Brussels, Belgium \\
\hline Benzothiazole & & & \\
\hline $\begin{array}{l}\text { Sulfenamide } \\
\text { (CBS) }\end{array}$ & & & \\
\hline $\begin{array}{l}\text { Di-Phenyl } \\
\text { Guanidine (DPG) }\end{array}$ & 2.0 & 4 & Flexsys, Brussels, Belgium \\
\hline
\end{tabular}

The materials were mixed in an internal mixer. Vulcanized specimens with a thickness of 2 $\mathrm{mm}$ were prepared for tensile tests, while vulcanized specimens with a thickness of $5 \mathrm{~mm}$ and a diameter of $50 \mathrm{~mm}$ were prepared for tribometer tests.

Tensile measurements were performed using an Instron tensile tester. The stress-strain relation for the two materials is depicted in Figure 1. The elastomer behaves nonlinearly at relatively 
high strains. The mechanical properties of the elastomer at high strains are a complex problem and are still the subject of several studies. Therefore, in the present study, the elastic moduli of the materials were defined at a strain of $2 \%$, assuming the material behaves linearly at that strain [35]. From the experimental results, the elastic moduli of the SBR-BR mix and the SBR material were $4.69 \pm 0.2 \mathrm{MPa}$ and $4.90 \pm 0.4 \mathrm{MPa}$, respectively.

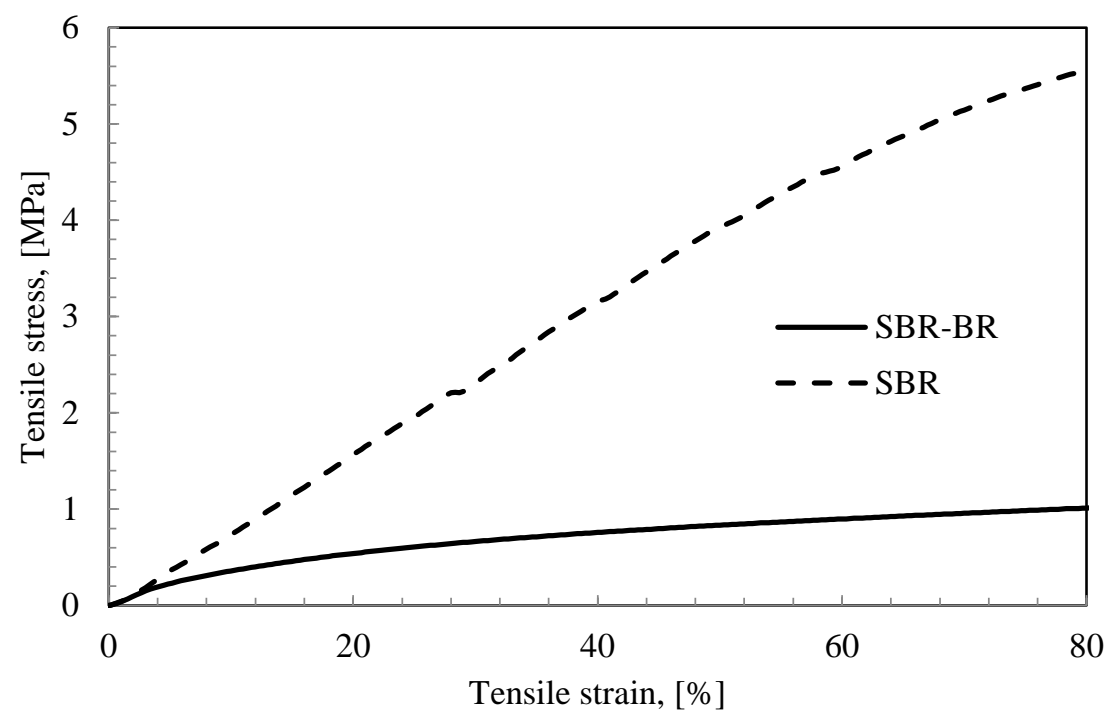

Figure 1. Stress-strain relationship of the materials listed in Table 1.

A Dynamic Mechanical Analyzer (DMA) was used to determine the dynamic properties of the materials. Creep compliances of the materials were determined using a Metravib Viscoanalyzer DMA+150 in shear mode, constant stress and at ambient temperature. Figure 2 shows that the creep compliance of the SBR-BR materials is higher than that of the SBR materials. The creep compliance results were fitted using a series of discrete exponential terms given by [36]:

$$
\phi(t)=\phi_{r}-\sum_{i=1}^{3} \phi_{i} \exp \left(\frac{-t}{\lambda_{i}}\right)
$$

where $\phi_{r}$ indicates the creep at a fully relaxed state, $t$ indicates time and $\lambda_{i}$ indicates the retardation times. As can be seen in Figure 2, the creep compliances of the materials are well described by the equation. The parameter values of the fit equation can be found in Table 2 for both materials. 


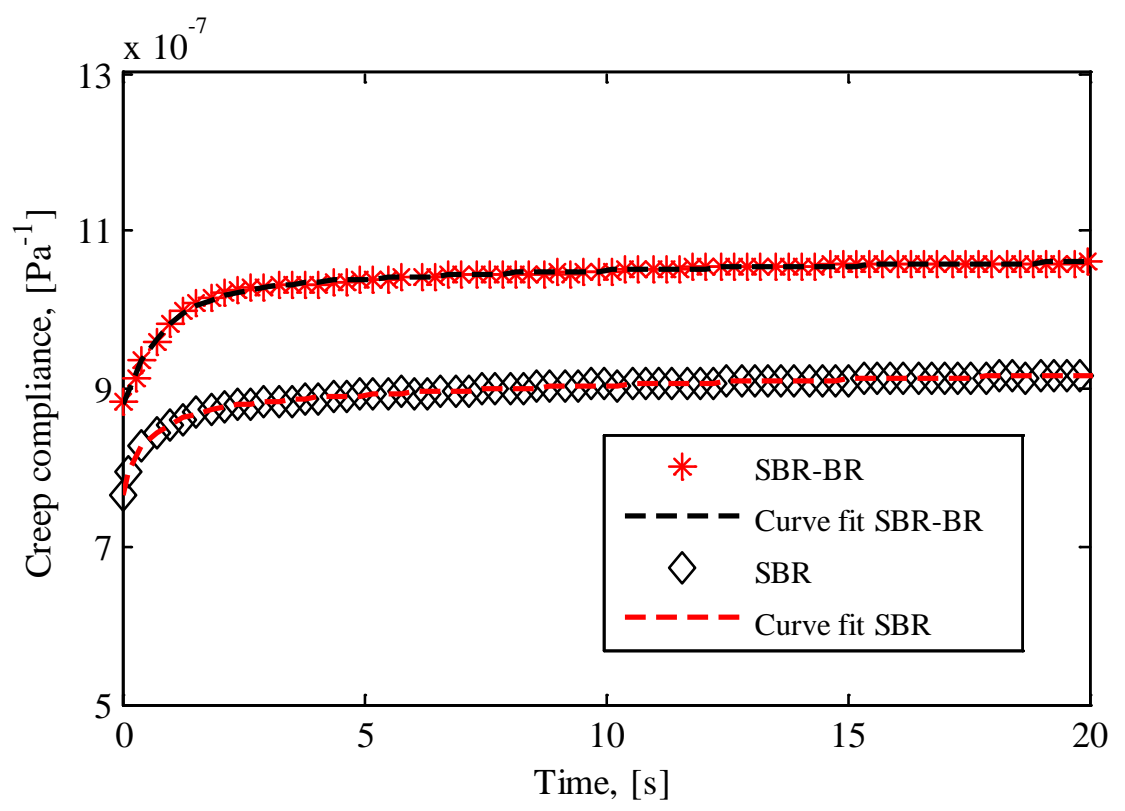

Figure 2. Experimental data and curve fitting of the creep compliances for both materials.

Table 2. Parameter values of the fit equation.

\begin{tabular}{lcc}
\hline & SBR-BR & SBR \\
\hline$\phi_{r}\left[\mathrm{~Pa}^{-1}\right]$ & $1.07 \times 10^{-06}$ & $9.26 \times 10^{-07}$ \\
$\phi_{1}\left[\mathrm{~Pa}^{-1}\right]$ & $1.42 \times 10^{-07}$ & $4.22 \times 10^{-08}$ \\
$\phi_{2}\left[\mathrm{~Pa}^{-1}\right]$ & $2.11 \times 10^{-07}$ & $6.64 \times 10^{-08}$ \\
$\phi_{3}\left[\mathrm{~Pa}^{-1}\right]$ & $2.87 \times 10^{-08}$ & $5.19 \times 10^{-08}$ \\
$\lambda_{1}[\mathrm{~s}]$ & 0.86 & 0.21 \\
$\lambda_{2}[\mathrm{~s}]$ & 6.79 & 0.94 \\
$\lambda_{3}[\mathrm{~s}]$ & 19.97 & 11.79 \\
\hline
\end{tabular}

\subsection{Methods}

A schematic illustration of the pin-on-disc tester used in the present study is depicted in Figure 3. It consists of a rotating elastomer disc and a spherically shaped pin. The total force of the pin on the elastomer surface consists of a static and a dynamic component, the static force is caused by the dead weight applied on the elastomer surface, while the dynamic force is caused by the inertia force of the counter surface frame, i.e., dead weight, beam, and counterweight during sliding. 


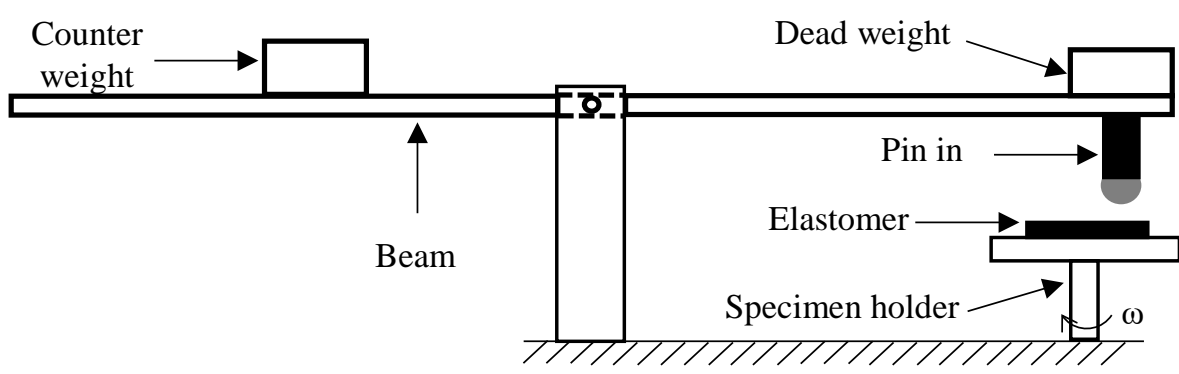

Figure 3. Pin-on-disc tribometer, schematically.

During sliding, oscillations can occur both in the normal and in the tangential (sliding) direction. When contact occurs between the elastomer surface and the counter surface, they oscillate together. These oscillations will be influenced by the dead weight mass $\left(m_{w}\right)$, the inertia of the frame $\left(m_{f}\right)$, the mechanical properties of the elastomer and the operating conditions of the test. In the appendix to this paper, a summary of the dynamical behavior of the system is given, based on the work of Setiyana, et al. [37].

For low sliding velocities, the oscillation will take place dominantly in the tangential direction. In this case, the effect of static and kinetic friction will be important. A study of stick-slip behavior when oscillation only occurs in the tangential direction has been done in a previous study [38]. For high sliding velocities, the normal direction oscillation of the elastomer surface is dominating the total dynamic response, meaning that the vibrations in tangential direction can be neglected. This study focuses on the macro scale irregularity of the wear track due to the normal oscillation of the system. The normal oscillation frequency of the system is defined as [37]:

$$
f_{n}=\frac{1}{2 \pi} \sqrt{\frac{k_{n}\left(1-\xi_{f}^{2}\right)}{m_{w}+m_{f}}}
$$

Where $f_{n}$ is the frequency of the counter surface system in the normal direction, $k_{n}$ is the normal stiffness of the elastomer, $\xi_{f}$ is the damping factor of the frame, $m_{w}$ is the dead weight mass and $m_{f}$ is the inertia of the frame. Since the materials used in the present study behave isotropicviscoelastic, the indentation depth $\delta$ can be estimated as [39]:

$$
\delta=\left(\frac{9 F_{n}^{2}}{16 E^{2} R} \bar{\phi}(t)\right)^{1 / 3}
$$


Where $\delta$ is the indentation depth, $F_{n}$ is the normal force, $E^{*}$ is the reduced elastic modulus, $R$ is the radius of the spherical counter surface, and $\bar{\phi}(t)$ is the normalized creep compliance. The stiffness in normal direction of the material is defined as the ratio of the normal force and the indentation depth:

$$
k_{n}=\frac{F_{n}}{\delta}
$$

The wave length $\left(\Delta x_{n}\right)$ of the wavy wear pattern is the ratio of the given sliding velocity and the normal frequency of the system, which is defined as:

$$
\Delta x_{n}=2 \pi v \sqrt{\frac{m_{w}+m_{f}}{k_{n}\left(1-\xi_{f}^{2}\right)}}
$$

Where $v$ is the sliding velocity. A detailed explanation about this analytical dynamic model for the set-up can be found in the Appendix.

The pin-on-disc tribometer was equipped with a ball with a radius of $12.5 \mathrm{~mm}$, sliding against an elastomer flat disc. The tests were performed under dry condition and at room temperature. Table 3 lists the operating conditions that were used in the present study. For the SBR-BR material, a different radius of the wear track was used, see test \#1 to test \#3 in Table 3 . The normal force and sliding velocity were varied for the SBR material. Due to the lack of a damper in the pin-on-disc tribometer set-up, the damping factor of the counter surface frame is neglected $\left(\xi_{n d}=0\right)$. The inertia mass of the frame in the present study is approximately 0.26 $\mathrm{Kg}$. Since the type and the dimension of the rigid ball are the same for all tests, the inertia mass of the counter surface frame $(m f)$ is the same for all tests.

Table 3. Operating conditions of pin-on-disc tests.

\begin{tabular}{l|cccccc}
\hline Test ID & $\# 1$ & $\# 2$ & $\# 3$ & $\# 4$ & $\# 5$ & $\# 6$ \\
\hline Disc material & SBR-BR & SBR-BR & SBR-BR & SBR & SBR & SBR \\
Normal force [N] & 2 & 2 & 2 & 3 & 5 & 5 \\
Velocity [m/s] & 0.2 & 0.2 & 0.2 & 0.2 & 0.2 & 0.3 \\
Radius of track [m] & 0.006 & 0.0125 & 0.019 & 0.012 & 0.012 & 0.012 \\
\hline
\end{tabular}

\section{Results and Discussion}

\subsection{Analytical results}


The analytical calculation was conducted with Eq. (1) - Eq. (5), in which the wave length depends on the mechanical properties of the material and the operating conditions used in the experiment, such as normal force and sliding velocity.

Figure 4 shows examples of analytical calculations for two types of materials with different elastic modulus, i.e., $5 \mathrm{MPa}$ and $10 \mathrm{MPa}$. The inertia mass of frame of $0.26 \mathrm{Kg} . \mathrm{m}^{2}$ and the radius of the spherical counter surface of $12.5 \mathrm{~mm}$ were used in the calculations. The sliding velocity was kept constant, $v=0.2 \mathrm{~m} / \mathrm{s}$, and the value of the normal force was varied. The creep compliance of the SBR material was used in these calculations. The results show that the wave length increases with increasing normal force. It is caused by the system oscillating with difficulty at a higher normal force so that the normal oscillation frequency reduces. As a result, the wave length increases when a constant sliding velocity is applied. At very high normal forces, the wave length increases marginally. On the other hand, a stiffer material shows a shorter wave length compared to the soft material. The stick-slip or wave motion often occurs in soft materials and diminishes in stiff materials [20, 40].

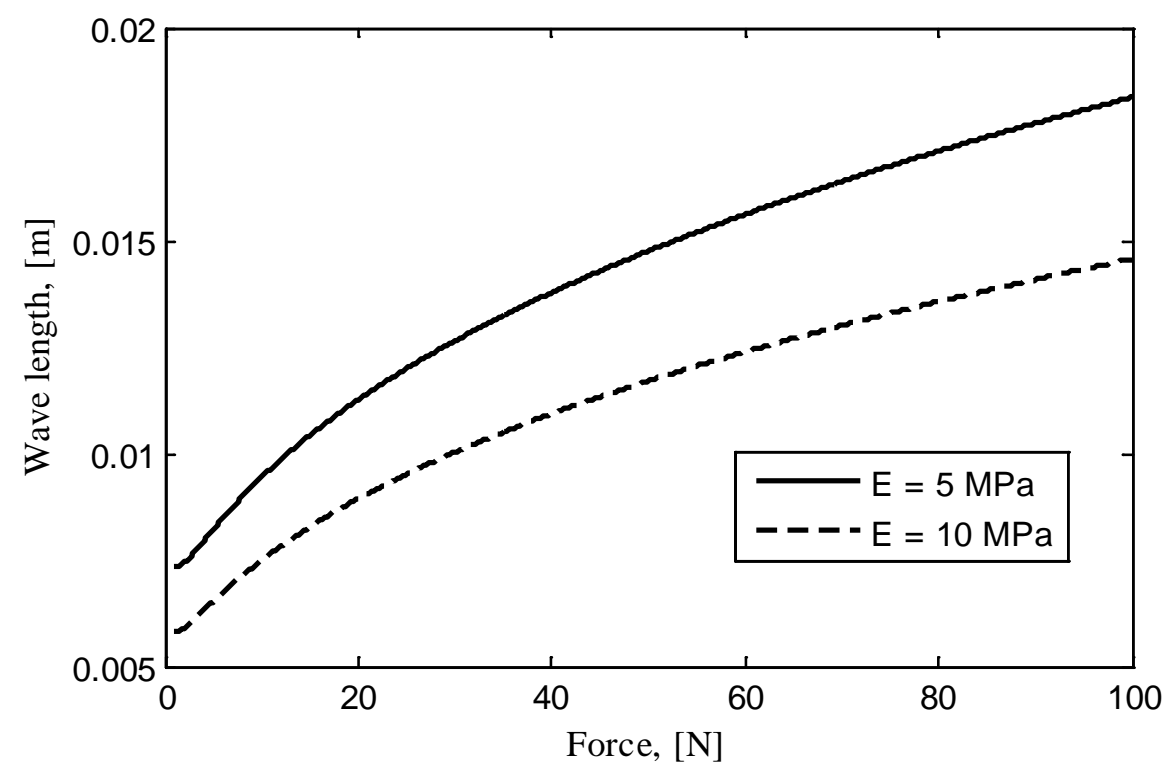

Figure 4. The wave length as a function of the normal force at a sliding velocity of $0.2 \mathrm{~m} / \mathrm{s}$.

The effect of sliding velocity on the wave length is depicted in Figure 5. The materials, inertia mass of frame and radius of the spherical counter surface used in these calculations are the same as the calculations in Figure 4. A constant normal force of $1 \mathrm{~N}$ was used, and the sliding velocity was varied. The results show that the wave length increases linearly with increasing sliding velocity. This can be explained by the fact that a longer traveling distance in one 
periodic oscillation can be achieved at a higher sliding velocity when a constant normal oscillation frequency is applied. As a result, a longer wave length is found, which is consistent with previous studies $[9,41]$. Moreover, a shorter wave length is found for the stiffer material because it reduces the normal oscillation frequency. The different wave lengths between both materials become more pronounced at a high sliding velocity.

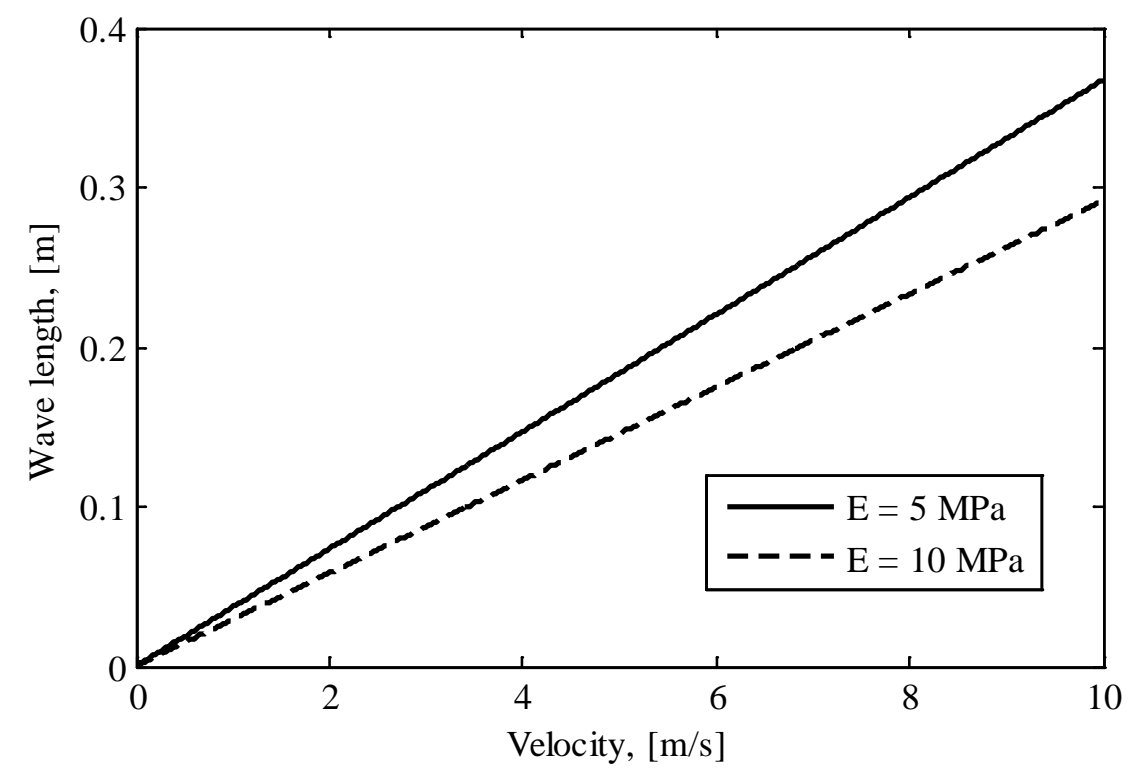

Figure 5. The wave length as a function of sliding velocity at a normal force of $1 \mathrm{~N}$.

\subsection{Experimental results}

Pin-on-disc tribometer experiments were carried out using several materials and under various operating conditions to validate the analytical model. The tests were stopped when the coefficient of friction reached a steady state phase. Figure 6 shows the wear track results of SBR-BR material under several operating conditions, i.e., test \#1 to test \#3. It is clearly seen that a wavy wear track is observed in test \#1, while the wear tracks in test \#2 and test \#3 are relatively smooth without a wave pattern. The number of wavy wear patterns on the SBR-BR surface in test \#1 is 5, with a wave length of approximately $7.54 \mathrm{~mm}$, see Figure 6. 


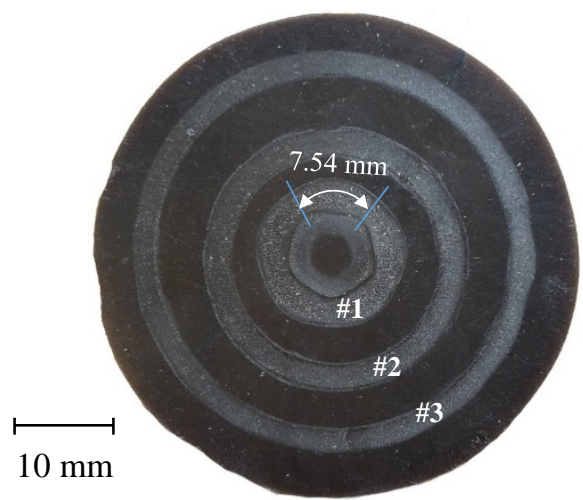

Figure 6. The wear tracks on the surface of SBR-BR material in test \#1 to test \#3.

The wave length of each test was calculated based on Eq. (1) - Eq. (5) using the operational conditions given in Table 3, together with the inertia mass of the counter surface frame and the mechanical properties of the materials. The creep compliances of the materials can be obtained by putting the parameter values of Table 2 into Eq. (1). The time used in the calculation is based on the contact time between the counter surface and the elastomer. Since the normal force and sliding velocity of test \#1 to test \#3 are the same, their normal oscillation frequencies are the same. As a result of the calculations, the wave length is $7.56 \mathrm{~mm}$ for those tests. This result is in agreement with the wave length which is observed in test \#1, see Figure 6. Although test \#2 and test \#3 have the same wave length, the wavy wear track is not observed on the SBRBR surface after those tests. This is because of the different locations of the waves between the different cycles, see Figure 7a. The aforementioned term 'cycle' means one revolution of the disc. When the locations of waves are at the same position in every cycle, the wavy wear track will be visible at the surface after the test. However, when the locations of the waves are different in each cycle, the wear track becomes relatively smooth after a large number of cycles, and the wavy wear track will not be visible, see Figure $7 \mathrm{~b}$.

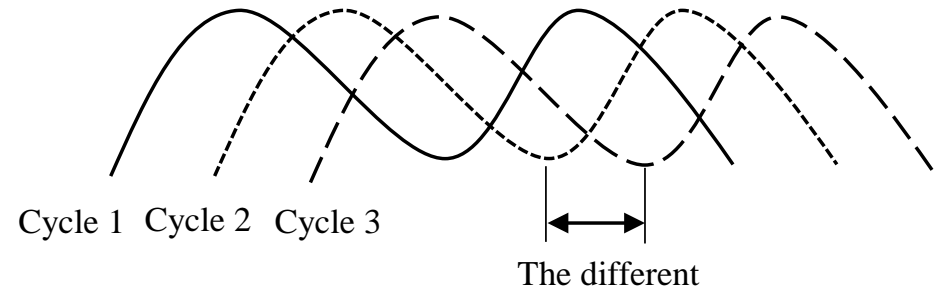

location of wave

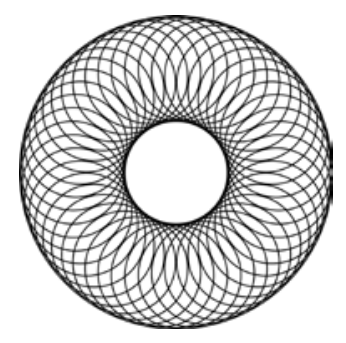

(b) 
Figure 7. (a) The waves at three different locations, (b) the final wear track for a large number of cycles when the locations of waves are different in each cycle, schematically.

The requirement of the occurrence of a wavy wear track is that the location of the waves has to be the same in each cycle. It means that the ratio between the circumferential distance of the wear track and the wave length has to be an integer. The number of wavy wear patterns in the circumferential of wear track can be expressed as:

$$
n=\frac{2 \pi r}{\Delta x_{n}}
$$

Where, $n$ is the number of the wavy wear patterns, $\Delta x_{n}$ is the wave length, and $r$ is the radius of the wear track.

By putting the calculated result of the wave length $(7.56 \mathrm{~mm})$ and the radius of the wear track given in Table 3 into Eq. (6), the number of wavy wear patterns in the circumferential direction in the wear track is $4.99 \approx 5,10.39$ and 15.80 in test \#1, test \#2 and test \#3, respectively. The number of wavy wear patterns in test \#1 was found to be an integer and in agreement with the experimental result, see Figure 6 . At the same time, the non-integer numbers of wavy wear patterns in the circumferential track were found in test \#2 and test \#3. This is the reason why they had smooth wear tracks at the end of the tribometer tests.

The effects of normal force and sliding velocity on the occurrence of a wavy wear track were investigated using SBR material, see Figure 8. It can be seen that the wavy wear track is not observed in test \#4, whereas it is visible in tests \#5 and \#6. The number of wavy wear patterns in test \#5 and test \#6 is 9 and 6, respectively. By using the same calculation procedure, the number of wave patterns in the circumferential direction of the wear track can be estimated for those tests. As can be seen from the results, the number of wave patterns in test \#4, test \#5 and in test \#6 is 9.65, $9.01 \approx 9$ and 6.01 $\approx 6$, respectively. These results are in good agreement with the experimental results, see Figure 8. 
(a)

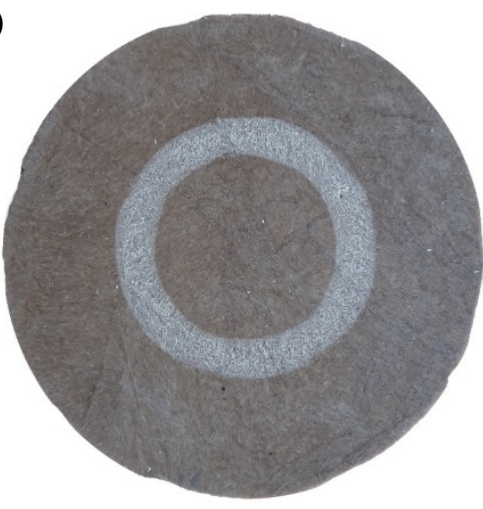

(b)

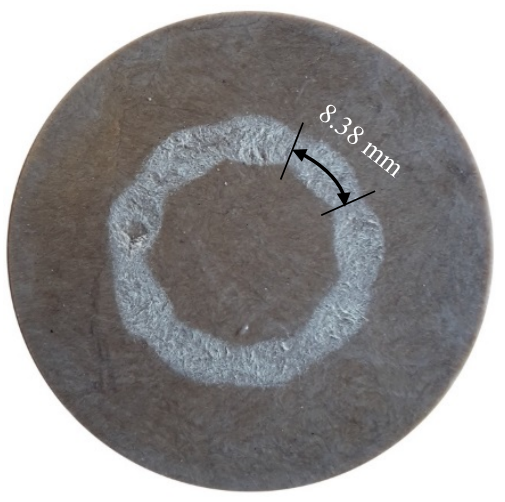

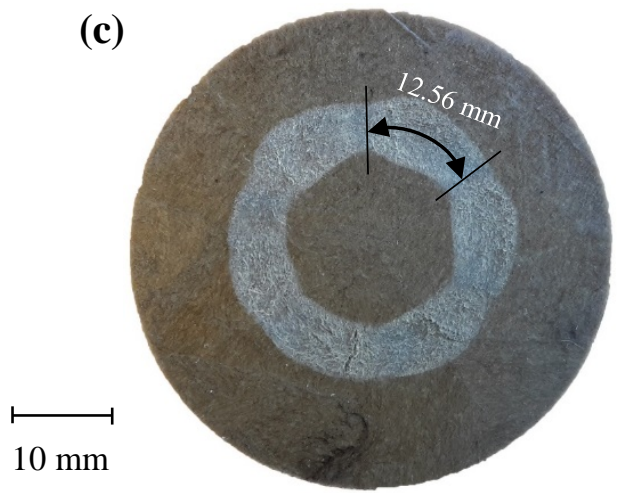

Figure 8. The wear tracks on the surface of SBR material in: (a) test \#4, (b) test \#5 and (c) test \#6.

The comparison between the analytical model and the experimental results for all materials is summarized in Table 4. It can be seen that the number of wavy wear patterns in the circumferential direction in test \#2, test \#3 and test \#4 is not an integer and, as a result, no wavy wear track is observed.

Table 4. The comparison between the analytical model and experimental results.

\begin{tabular}{|c|c|c|c|c|c|c|}
\hline $\begin{array}{l}\text { Tests } \\
\text { ID }\end{array}$ & $\begin{array}{c}\text { Force } \\
{[\mathrm{N}]}\end{array}$ & $\begin{array}{c}\text { Velocity } \\
{[\mathrm{m} / \mathrm{s}]}\end{array}$ & $\begin{array}{l}\text { Radius of the } \\
\text { track [mm] }\end{array}$ & $\begin{array}{c}\Delta x_{n}[\mathrm{~mm}] \\
\text { (model) }\end{array}$ & $\begin{array}{c}\Delta x_{n}[\mathrm{~mm}] \\
\text { (experiment) }\end{array}$ & $\frac{2 \pi r}{\Delta x_{n}(\text { model })}$ \\
\hline$\# 1$ & 2 & 0.2 & 6 & 7.56 & 7.54 & $4.99 \approx 5$ \\
\hline$\# 2$ & 2 & 0.2 & 12.5 & 7.56 & - & 10.39 \\
\hline \#3 & 2 & 0.2 & 19 & 7.56 & - & 15.80 \\
\hline$\# 4$ & 3 & 0.2 & 12 & 7.81 & - & 9.65 \\
\hline$\# 5$ & 5 & 0.2 & 12 & 8.37 & 8.38 & $9.01 \approx 9$ \\
\hline \#6 & 5 & 0.3 & 12 & 12.55 & 12.56 & $6.01 \approx 6$ \\
\hline
\end{tabular}


It is shown that the analytical model that predicts the occurrence of a wavy wear track is in good agreement with the experimental results. The wave length can be estimated based on the mechanical properties of the material, the inertia mass of the counter surface frame and the operating conditions used in the experiments, such as normal force and sliding velocity. In a previous study, the stick-slip motion is likely to occur for small normal force and large sliding velocity [32]. However, the occurrence of a wavy wear track not only depends on the stick-slip motion of the system but also on the circumferential length of the wear track, as seen in test \#1 to test \#3.

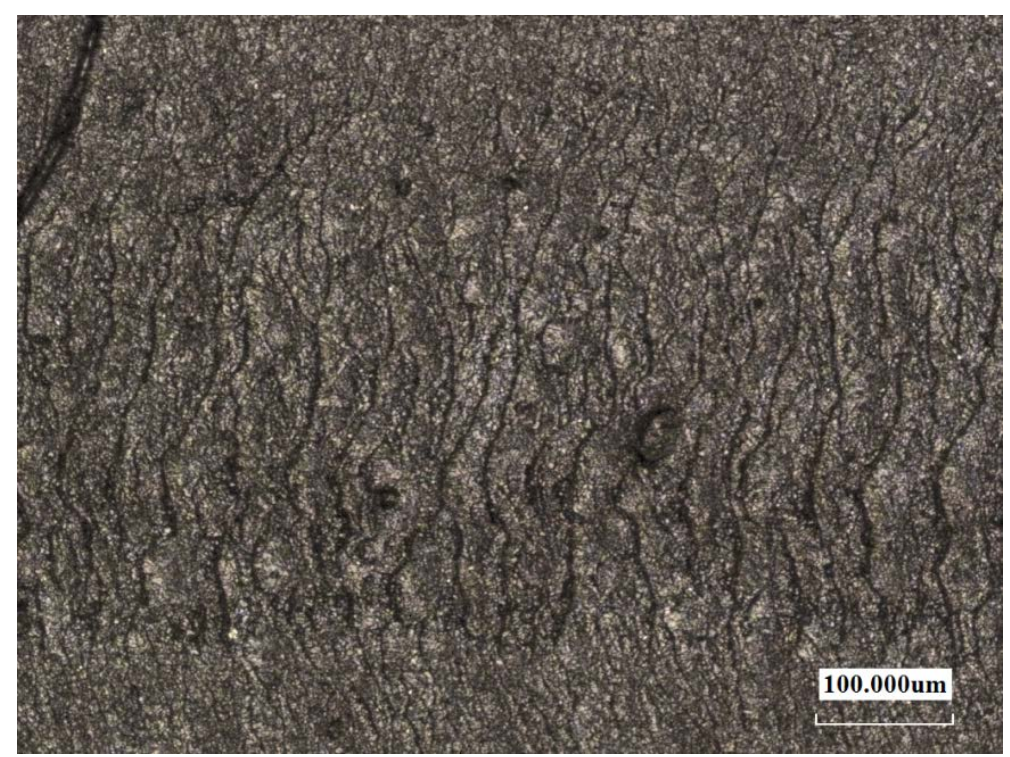

Figure 9. Crack patterns on the SBR-BR surface in test \#2.

Figure 9 shows an image of wear surface on the SBR-BR material in test \#2. Although a wavy wear track is not visible in this test, crack patterns are found in the wear track. This phenomenon was also reported by many other studies, see for instance [9-10, 22, 30, 42-43]. These crack patterns can be associated with the wave of the system during sliding contact. Since the locations of the waves are different for each cycle, the small crack patterns are formed after a large number of cycles, see Figure 7b. For the SBR material, no specific wear pattern is formed on the wear surface. This is caused by the presence of fibers on the wear surface preventing the specific crack pattern, which is consistent with a previous study [44]. For a fiber reinforced elastomer without any filler (such as silica and carbon black), the rigid counter surface is mainly in contact with the fibers in the steady state phase [45]. 
Based on the present study, a wavy wear track can be prevented by adjusting several parameters like the radius of the wear track, the sliding velocity and the normal force. The wavy wear track may generate inaccuracy of friction and wear results of pin-on-disc tribometer experiments. By preventing a wavy wear track, this inaccuracy can be avoided. In the real engineering application, not only a macro-wave has to be prevented, but also a micro-wave (crack pattern) as well. Therefore, the best way to prevent both is by adjusting the dynamics of the system in which the elastomer is operating through the application of a damper system, for instance.

\section{Conclusion}

The wavy wear track of elastomers as a function of several operating conditions was studied. A rigid indenter ball and two types of elastomers made of Styrene-Butadiene Rubber (SBR) and Butadiene Rubber (BR) were used. The conclusions of this study can be summarized as follows:

1. The oscillations may occur in the system during sliding friction that can cause a macro (wavy wear track) and micro (crack pattern) wear pattern on the elastomer surface.

2. The wavy wear track can be prevented by adjusting the mechanical properties of the elastomer, the operational conditions (such as sliding velocity and force), the inertia mass of the system, and the circumferential length of the wear track. While the crack pattern may be prevented by adding a damper to the system.

3. An analytical model was developed to predict the occurrence of a wavy wear track, and it shows good agreement with the experimental results.

\section{Acknowledgment}

This research forms part of the Research Programme of the Dutch Polymer Institute DPI, Project \#782.

\section{Appendix [37]}

Figure A.1 shows the physical model that represents an elastomer surface in contact with a counter surface. It consists of an oscillating elastomer mass $\left(m_{e}\right)$ with stiffness and damping in tangential $\left(k_{t}, c_{t}\right)$ as well as in normal direction $\left(k_{n}, c_{n}\right)$. The acting forces in the contacting surfaces are the driving force $\left(F_{d}\right)$ of the elastomer, the tangential contact force $\left(F_{t}\right)$ and the 
normal contact force $\left(F_{n}\right)$. During sliding friction, there are two possibilities of contact interaction between the elastomer surface and the counter surface, i.e., separation and unification. The separation means no contact between the elastomer surface and the counter surface, see Figure A.1 (a). In this case, the oscillating mass of the elastomer $\left(m_{e}\right)$ oscillates without the mass of the counter surface system $\left(m_{s}\right)$. The unification interaction means that the elastomer surface and the counter surface are unified, and they oscillate together. The unified mass $(M)$ is an aggregate of the oscillating elastomer mass and the inertia mass of the counter surface system $\left(M=m_{e}+m_{s}\right)$, see Figure A.1 (b). The oscillating elastomer mass is assumed to be very small compared to the inertia mass of the counter surface frame, so that it can be neglected. Thus, the oscillating unified mass can be written as $M \approx m_{s}=m_{w}+m_{f}$, where $m_{w}$ is the dead weight mass and $m_{f}$ is the inertia mass of the counter surface frame. An equivalent damping is denoted by $c_{n d}$, which is a combination of the elastomer and the apparatus dashpot. Based on Figure A.1 (b), the equation of motion in tangential direction is given in Eq. (A.1) by involving the tangential force $\left(F_{t}\right)$. Where, $v$ is the sliding velocity and $t$ is time. While the equation of motion in normal direction is given in Eq. (A.2).

$$
\begin{gathered}
m_{e u} \ddot{x}+c_{t} \dot{x}+k_{t} x=k_{t} v t+c_{t} v-F_{t} \\
M \ddot{y}+c_{n d} \dot{y}+k_{n} y=0
\end{gathered}
$$

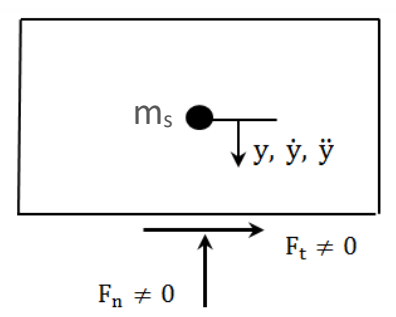

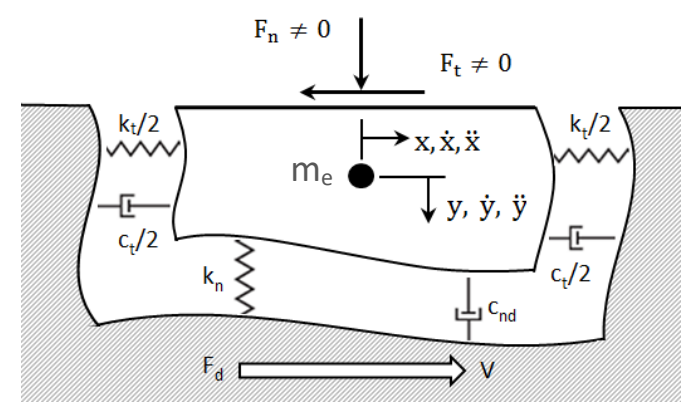

(a)

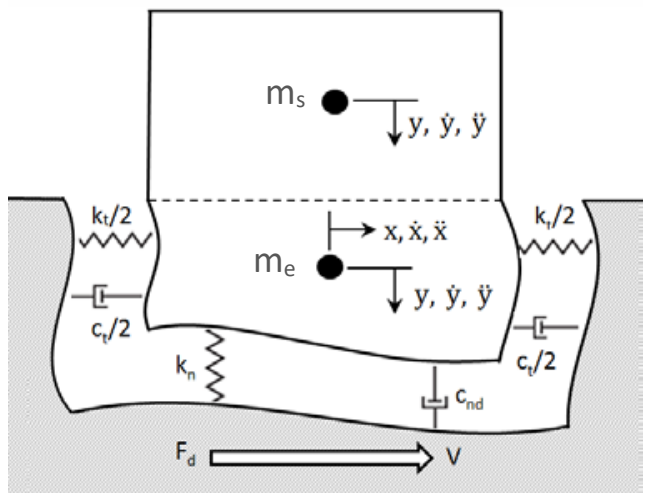

(b)

Figure A.1. The interaction during sliding contact between an elastomer surface and a counter surface, (a) separation, (b) unification [37]. 
For a high driving velocity, the elastomer surface dominantly oscillates in the normal direction, meaning that the oscillation in tangential direction can be neglected. The natural frequency in the normal oscillation for the undamped system $\left(\omega_{n}\right)$ can be defined as follows:

$$
\omega_{n}=\sqrt{\frac{k_{n}}{M}}
$$

When the system uses a dashpot, a damped natural frequency $\left(\omega_{d}\right)$ is given by:

$$
\omega_{d}=\omega_{n} \sqrt{1-\xi_{f}^{2}}
$$

The normal oscillation period of the system $\left(T_{n}\right)$ can be found by using the following equation:

$$
T_{n}=2 \pi \omega_{d}
$$

By putting Eq. (A.3) and Eq. (A.4) into Eq. (A.5), the normal oscillation period can be expressed as follows:

$$
T_{n}=2 \pi \sqrt{\frac{M}{k_{n}\left(1-\xi_{f}^{2}\right)}}
$$

The periodic displacement $\left(\Delta x_{n}\right)$ in tangential direction due to normal oscillation is:

$$
\Delta x_{n}=v T_{n}=2 \pi v \sqrt{\frac{M}{k_{n}\left(1-\xi_{f}^{2}\right)}}
$$

\section{References}

1. B. Lorenz, Y.R. Oh, S.K. Nam, S.H. Jeon, B.N.J. Persson, Rubber friction on road surfaces: Experiment and theory for low sliding speeds, J. Chem. Phys. 142 (2015) 194701.

2. G. Bódai, T.J. Goda, Friction Force Measurement at Windscreen Wiper/Glass Contact, Tribol. Lett. 45 (2012) 515-523.

3. L.H. Zhao, X.B. Wei, X.G. Li, The Analysis on the Tribological Properties of CR/EPDM Blends with Chrome-Plated Steel under Dry Sliding, Adv. Mater. Res. 774776 (2013) 99-102.

4. $\quad$ L.I. Farfán-Cabrera, E.A. Gallardo-Hernandez, J.B. Pascual-Francisco, C.D. ResendizCalderon, C.S. de la Rosa, Experimental method for wear assessment of sealing elastomers, Polym. Test. 53 (2016) 116-121.

5. M. Khafidh, N.V. Rodriguez, M.A. Masen, D.J. Schipper, The dynamic contact area of elastomers at different velocities, Tribology - Mater. Surf. Interfaces 10 (2016) 70-73. 
6. M. Mokhtari, D.J. Schipper, T.V. Tolpekina, On the Friction of Carbon Black- and Silica-Reinforced BR and S-SBR Elastomers, Tribol. Lett. 54 (2014) 297-308.

7. $\quad$ N.G. Payne, R.G. Bayer, Friction and wear tests for elastomers, Wear 150 (1991) 6777.

8. M. Mofidi, E. Kassfeldt, B. Prakash, Tribological behaviour of an elastomer aged in different oils, Tribol. Int. 41 (2008) 860-866.

9. Y. Fukahori, H. Yamazaki, Mechanism of rubber abrasion. Part I: Abrasion pattern formation in natural rubber vulcanizate, Wear 171 (1994) 195-202.

10. Z. Mané, J.-L. Loubet, C. Guerret, L. Guy, O. Sanseau, L. Odoni, L. Vanel, D.R. Long, P. Sotta, A new rotary tribometer to study the wear of reinforced rubber materials, Wear 306 (2013) 149-160.

11. W. Molnar, M. Varga, P. Braun, K. Adam, E. Badisch, Correlation of rubber based conveyor belt properties and abrasive wear rates under 2- and 3-body conditions, Wear 320 (2014) 1-6.

12. S. Maegawa, F. Itoigawa, T. Nakamura, Dynamics in sliding friction of soft adhesive elastomer: Schallamach waves as a stress-relaxation mechanism, Tribol. Int. 96 (2016) 23-30.

13. K. Nakano, S. Maegawa, Safety-design criteria of sliding systems for preventing friction-induced vibration, J. Sound Vib. 324 (2009) 539-555.

14. A. Schallamach, How does rubber slide?, Wear 171 (1971) 301-312.

15. B. Best, P. Meijers, A.R. Savkoor, The formation of Schallamach waves, Wear 65 (1981) 385-396.

16. B.N.J. Persson, Elastic instabilities at a sliding interface, Phys. Rev. B 63 (2001) 104101.

17. M. Barquins, Energy dissipation in Schallamach waves, Wear 91 (1983) 103-110.

18. M. Barquins, Friction and wear of rubber-like materials, Wear 160 (1993) 1-11.

19. A. Koudine, M. Lambert, M. Barquins, Some new experimental results on the Schallamach waves propagation by space-time analysis, Int. J. Adhes. Adhes. 17 (1997) 359-363.

20. C.J. Rand, A.J. Crosby, Insight into the periodicity of Schallamach waves in soft material friction, Appl. Phys. Lett. 89 (2006) 261907.

21. A. Schallamach, Abrasion of rubber by a needle, J. Polym. Sci. 9 (1952) 385-404.

22. A. Schallamach, Friction and abrasion of rubber, Wear 1 (1958) 384-417.

23. A.G. Thomas, Factors influencing the strength of rubbers, J. Polym. Sci. Polym. Symp. 48 (1974) 145-157.

24. D. Champ, E. Southern, A. Thomas, Fracture mechanics applied to rubber abrasion, in: L.-H. Lee (Eds.), Advances in Polymer Friction and Wear, Springer, Boston, 1974, pp. 133-144.

25. H. Liang, Y. Fukahori, A.G. Thomas, J.J.C. Busfield, Rubber abrasion at steady state, Wear 266 (2009) 288-296.

26. V. Coveney, C. Menger, Initiation and development of wear of an elastomeric surface by a blade abrader, Wear 233-235 (1999) 702-711.

27. Y. Fukahori, H. Liang, J.J.C. Busfield, Criteria for crack initiation during rubber abrasion, Wear 265 (2008) 387-395.

28. Y. Fukahori, H. Yamazaki, Mechanism of rubber abrasion, Wear 178 (1994) 109-116.

29. Y. Fukahori, H. Yamazaki, Mechanism of rubber abrasion part 3: how is friction linked to fracture in rubber abrasion?, Wear 188 (1995) 19-26.

30. Y. Fukahori, H. Yamazaki, Mechanism of rubber abrasion: Part 2. General rule in abrasion pattern formation in rubber-like materials, Wear 178 (1994) 109-116. 
31. M. Barquins, R. Courtel, Rubber friction and the rheology of viscoelastic contact, Wear 32 (1975) 133-150.

32. S. Maegawa, K. Nakano, Mechanism of stick-slip associated with Schallamach waves, Wear 268 (2010) 924-930.

33. A.A. Koudine, M. Barquins, Formation of micro-ridges on the surface of Schallamach waves propagating in the contact area between a moving rubber sample and a glass lens, J. Adhes. Sci. Technol. 10 (1996) 951-961.

34. R. Rauline, (to Compagnie Generale des Establissements Michelin) EU 0501227 (1992).

35. C. Hintze, R. Boldt, S. Wiessner, G. Heinrich, Influence of processing on morphology in short aramid fiber reinforced elastomer compounds, J. Appl. Polym. Sci. 130 (2013) 1682-1690.

36. J.D. Ferry, Viscoelastic Properties of Polymers, second ed., John Wiley and sons, New York (1980).

37. B. Setiyana, R. Ismail, J. Jamari, D.J. Schipper, An analytical study of the wear pattern of an abraded rubber surface: The interaction model, Tribology - Mater. Surf. Interfaces (2018) 1-7.

38. B. Setiyana, R. Ismail, J. Jamari, D.J. Schipper, Stick-Slip Behavior of a Viscoelastic Flat Sliding along a Rigid Indenter, Tribol. Online 11 (2016) 512-518.

39. N.V. Rodriguez, M.A. Masen, D.J. Schipper, A contact model for OrthotropicViscoelastic Materials, Int. J. Mech. Sci 74 (2013) 91-98.

40. S. Maegawa, K. Nakano, Dynamic behaviors of contact surfaces in the sliding friction of a soft material, J. Adv. Mech. Des. Syst. 1 (2007) 553-561.

41. K. Nakano, S. Maegawa, Occurrence limit of stick-slip: dimensionless analysis for fundamental design of robust-stable systems, Lubrication Science 22 (2010) 1-18.

42. V.G. Kopchenkov, Modeling elastomer wear under the effects of solid particles at an angle to the surface, J. Frict. Wear 38 (2017) 162-167.

43. A.H. Muhr, A.D. Roberts, Rubber abrasion and wear, Wear 158 (1992) 213-228.

44. N. Wada, Y. Uchiyama, Friction and wear of short-fibre-reinforced rubber composites under various sliding speeds and loads, Wear 162-164 (1993) 930-938.

45. M. Khafidh, D.J. Schipper, M.A. Masen, N. Vleugels, J.W.M. Noordermeer, Friction of Short-cut Aramid Fiber Reinforced Elastomer, Proceedings of the $6^{\text {th }}$ World Tribology Congress (2017). 